\title{
BMJ Open Anlotinib combined with SOX regimen (S1 (tegafur, gimeracil and oteracil porassium capsules) + oxaliplatin) in treating stage IV gastric cancer: study protocol for a single-armed and single- centred clinical trial
}

Juan Wang, ${ }^{1}$ Dong Xue Wu, ${ }^{2}$ Lu Meng, ${ }^{2}$ Gang Ji (D) ${ }^{1}$

To cite: Wang J, Wu DX, Meng L, et al. Anlotinib combined with SOX regimen (S1 (tegafur, gimeracil and oteracil porassium capsules) + oxaliplatin) in treating stage IV gastric cancer: study protocol for a single-armed and singlecentred clinical trial. BMJ Open 2020;10:e034685. doi:10.1136/ bmjopen-2019-034685

- Prepublication history and additional material for this paper are available online. To view these files, please visit the journal online (http://dx.doi org/10.1136/bmjopen-2019034685).

Received 01 October 2019 Revised 24 April 2020 Accepted 01 May 2020

A Check for updates

(C) Author(s) (or their employer(s)) 2020. Re-use permitted under CC BY-NC. No commercial re-use. See rights and permissions. Published by BMJ.

${ }^{1}$ Department of Digestive Surgery, Xi Jing Hospital, Fourth Military Medical University,

Xi'an, Shaanxi, China

${ }^{2}$ Medical Department, Chia Tai Tianqing Pharmaceutical Group Co Ltd, Lianyungang, Jiangsu, China

Correspondence to

Dr Gang Ji;

jigangfmmu@163.com

\section{ABSTRACT}

Introduction Anlotinib hydrochloride is a multitargeted receptor tyrosine kinase inhibitor that targets angiogenesis-related kinases and has already showed good safety and efficacy in some solid tumours. However, evidence on the safety and feasibility of anlotinib in patients with stage IV gastric cancer is scarce.

Methods and analysis This study is a single-armed and single-centred clinical study being designed to include 150 patients of stage IV gastric cancer. The patients' demographics, pathological characteristics, test results of blood, biochemistry and tumour markers before and after medication, disease-free survival and overall survival will be collected and analysed. The primary and main efficacy outcomes are objective response rate, progression-free survival, disease control rate and overall survival. The secondary efficacy outcome is safety indicator including the incidence of adverse drug reactions and adverse events after administration.

Ethics and dissemination Ethics approval has been obtained from the Ethics Committee at the First Affiliated Hospital (Xijing Hospital) of Fourth Military Medical University (KY20192111-F-1). The results of this study will be disseminated at several research conferences and as published articles in peer-reviewed journals.

Trial registration number ChiCTR1900026291

(registration date: 29 September 2019).

\section{INTRODUCTION}

Gastric cancer is currently the fourth most common malignancy worldwide with the second highest mortality rate. ${ }^{1}$ There are 952000 new cases in the world every year, with $73.5 \%$ in Asia and $47 \%$ in China. The most recent analytical data in $2012^{2}$ revealed that the incidence of gastric cancer in China ranked the second most common $(36.21 / 100$ 000 ), and the mortality rate was the third highest $(25.88 / 100000)$. In addition to the high incidence rate and recurrence rate, the most important reason for the high mortality
Strengths and limitations of this study

- First clinical trial that will provide evidence on anlotinib in stage IV gastric cancer.

- A large number of 150 patients with stage IV gastric cancer will be enrolled.

- Main efficacy outcomes are objective response rate, progression-freesurvival, disease control rate and overall survival.

- Secondary efficacy outcome is the safety indicator.

- Limitations are single-centre and single-arm study without control group.

rate of gastric cancer is that most patients have late-stage disease when they are diagnosed. However, chemotherapy has very limited therapeutic effect on advanced gastric cancer. ${ }^{3}$ In addition, advanced gastric cancer usually cannot be treated surgically due to invasion of the pancreas encircling large blood vessels or distant metastasis. Moreover, about $50 \%$ of localised lesions cannot be radically resected, and the surgical cure rate is still low. At present, chemotherapy and targeted therapy are the main treatments for advanced gastric cancer. ${ }^{4}$ Compared with optimal supportive care, standardised chemotherapy and targeted therapy prolong survival, relieve clinical symptoms and improve quality of life. ${ }^{5}$

In recent years, molecular targeted therapy has been highly effective with low toxicity in treating cancers such as breast, colorectal, thyroid and non-small cell lung cancers, and its combination with chemotherapy for advanced cancer has become a popular research topic. ${ }^{6-11}$ There is currently no standardised therapeutic option for advanced gastric cancer, and the treatment protocol varies globally. A large-scale retrospective 
study shows that patient baseline status is a single independent factor affecting survival rate among patients with late-stage gastric cancer. ${ }^{12}$ In addition, distant metastatic sites (especially peritoneum and liver), two or more metastases and low protein levels are risk factors for poor prognosis. $^{12}$

Protein tyrosine kinase (PTK) signalling pathway is involved in the proliferation, differentiation and migration of tumour cells. ${ }^{13}$ Interfering with or blocking the tyrosine kinase pathway can be used to influence the growth of tumour cells. Therefore, screening for PTK inhibitors has become a new method for discovering anti-tumour drugs. Anlotinib hydrochloride is a multitargeted receptor tyrosine kinase inhibitor that targets angiogenesis-related kinases such as vascular endothelial growth factor receptors 1,2 and 3 (VEGFR1/2/3), fibroblast growth factor receptors 1, 2 and 3 (FGFR1/2/3) and other tumor-associated kinases involved in cell proliferation. ${ }^{14}$ These tumour-associated kinases such as plateletderived growth factor alpha/beta $(\mathrm{PDGFR} \alpha / \beta)$, c-Kit and Ret are significantly inhibited by anlotinib ${ }^{15}$ and the angiogenesis kinase inhibition spectrum (eg, Met, FGFR1/2/3) is broader. ${ }^{16}$ Anlotinib hydrochloride also has significant inhibitory activities against some kinase targets that are under investigation such as Aurora-B, colony-stimulating factor one receptor, and discoidin domain receptor $1 .{ }^{17}$ Moreover, anlotinib hydrochloride has significant inhibitory activity against multiple kinase mutants such as PDGFR $\alpha$, c-Kit, c-Met and epidermal growth factor receptor and the inhibitory activity against mutants is even stronger than that of the wild-type. ${ }^{18}$ Therefore, the efficacy and safety of anti-angiogenic drugs (eg, anlotinib hydrochloride) combined with chemotherapy for advanced gastric cancer are worth exploring and researching to provide more evidence and guidance for clinical practice.

\section{METHODS AND ANALYSIS \\ Study design}

This study is a single-armed and single-centred clinical study.

\section{Inclusion and exclusion criteria}

The inclusion criteria are as follows. (1) The patients provided written informed consent form to participate in the study. (2) Patients are diagnosed with stage IV gastric adenocarcinoma by biopsy/pathology (any T, N, M1, with extra-gastric measurable lesions including unresectable locally advanced tumours, advanced tumours by R1 or R2 resection and recurrence or metastasis status post R0 resection. (3) Patients are 18 years of age or older with an Eastern Cooperative Oncology Group (ECOG) performance status score of 0 to 2 points. (4) Functions of the major organs meet the following criteria within 7 days before treatment: routine examination standard (without blood transfusion within 14 days): haemoglobin $\geq 90 \mathrm{~g} / \mathrm{L}$, absolute neutrophil count $\geq 1.5 \times 109 / \mathrm{L}$, platelet $\geq 80 \times 109 / \mathrm{L}$; and chemistry panel test meet the following criteria: total bilirubin (TBIL) $\leq 1.5$ times the upper limit of normal (ULN), alanine aminotransferase (ALT) and aspartate aminotransferase (AST) $\leq 2.5 \times$ ULN or if complicated with liver metastasis then ALT and AST $\leq 5 \mathrm{x}$ ULN, serum creatinine $\leq 1.5 \times$ ULN or creatinine clearance $\geq 60 \mathrm{~mL} / \mathrm{min}$. (5) Women of childbearing age agree to use contraceptives (eg, intrauterine devices, contraceptives or condoms) during the study period and within the first 6 months after the study; having a negative serum or urine pregnancy test 7 days prior to study enrolment and are non-lactating patients. Male candidates agree to use contraception during the study period and within the first 6 months after the study.

The exclusion criteria were as follows. (1) Patients who were previously treated with anlotinib. (2) Patients who were previously treated with VEGFR-tyrosine kinase inhibitors such as sunitinib, sorafenib, famitinib, apatinib, and regorafenib. (3) Patients currently suffering from or who previously had other malignant tumours within 5 years, except cervical cancer in situ, non-melanoma skin cancer and superficial bladder tumours (Ta (non-invasive tumour), Tis (in situ carcinoma) and T1 (tumourinfiltrating basement membrane)). (4) Patients received systemic anti-tumour therapy including cytotoxic therapy, signal transduction inhibitors and immunotherapy 4 weeks prior to enrolment or during the study or who were treated with mitomycin $\mathrm{C}$ in the 6 weeks before study. Patients received extended-filed radiation therapy in the 4 weeks before enrolment or involved field radiation therapy in the 2 weeks prior to enrolment. (5) Patients with unresolved toxic effects above Grade 1 Common Terminology Criteria for Adverse Events (CTCAE) (4.0) due to prior treatment, but not including alopecia and oxaliplatin-induced neurotoxicity that was lower than Grade 2. (6) Patients with any sign or history of haemorrhage, regardless of severity, with any bleeding or bleeding episodes $\geq$ CTCAE Grade 3 within 4 weeks prior to enrolment; or with unhealed wounds, ulcers or fractures. Patients with a clear possibility of gastrointestinal bleeding, including the following conditions: local active ulcer lesions, and faecal occult blood 2+; patients with a history of melena, haematemesis, faecal occult blood $1+$ in the past 2 months and patients with gastric tumour primary lesions without surgical resection, and considered to be at high risk of having gastrointestinal bleeding by main research staff. (7) Medical conditions that affected ability to take oral medication (eg, inability to swallow, chronic diarrhoea). (8) Patients with pleural effusion or ascites, causing respiratory symptoms ( $\geq$ CTCAE Grade 2 dyspnoea (level 2 dyspnoea refers to shortness of breath during low-intensity physical activity, affecting instrumental activities of daily living). (9) Patients with any of the following severe and/or uncontrolled diseases: taking one antihypertensive medication but still having unsatisfactory blood pressure (systolic blood pressure $\geq 150 \mathrm{~mm} \mathrm{Hg}$, diastolic blood pressure $\geq 100 \mathrm{~mm} \mathrm{Hg}$ ); suffering from Grade 1 or above myocardial ischaemia 
or myocardial infarction, malignant arrhythmia and $\geq$ Grade 2 congestive heart failure according to New York Heart Association classification; active or uncontrolled severe infection ( $\geq$ CTCAE Grade 2 infection); cirrhosis, decompensated liver disease or active hepatitis; renal failure requiring haemodialysis or peritoneal dialysis; history of immunodeficiency including HIV-positive or other acquired, congenital immunodeficiency diseases or a history of organ transplantation; poorly controlled diabetes (fasting blood glucose $(\mathrm{FBG})>10 \mathrm{mmol} / \mathrm{L}$ ); urinalysis indicating urine protein $\geq++$ and confirmed $>1.0 \mathrm{~g}$ of 24 hours urine protein; seizures that require treatment; history of psychotic medication abuse and are unable to quit; mental disorder; brain metastases with neurological symptoms or symptoms controlled for less than 2 months. (10) Patients who had major surgical treatment, open biopsy or significant traumatic injury within 28 days before enrolment. (11) Arterial/venous thrombosis caused events that occurred within 6 months such as cerebrovascular accidents (including transient ischaemic attacks), deep vein thrombosis and pulmonary embolism. (12) Patients who participated in other clinical trials of anti-tumour medications or are undergoing other clinical trials within 4 weeks.

The dropping out criteria is if chemotherapy or experimental drug treatments other than the presenting protocol is used during the trial.

The terminating study criteria are as follows: (1) Progressive disease (PD) will be determined based on efficacy evaluation criteria or considered by tumour markers, blood test and image test including enhanced CT or MRI of the chest, abdomen and pelvis. (2) Grade 3 or higher malignant pleural effusion or pneumothorax occur according to the National Cancer Institute (NCI) CTCAE 4.0 grading standard. (3) Interim analysis show that the medications lacked efficacy. (4) The researchers/ study clinicians consider that the treatment should be terminated from the perspective of the best benefit of the patient. (5) Intolerable adverse reactions or serious adverse events are confirmed by the investigators. (6) Patients with poor compliance and those taking medications other than $80 \%$ to $120 \%$ of the dose. (7) Patients who voluntarily withdrew informed consent. (8) Patients who use other anti-tumour drugs (eg, chemotherapy, targeted therapy or biological agents) that affect efficacy judgement. (9) Patients with unexpected pregnancy. (10) Death of the patient.

\section{Dosage regimen}

Anlotinib hydrochloride capsules are taken orally (before breakfast) once a day, $12 \mathrm{mg}$ each time, 2 weeks on and 1 week off; $\mathrm{S} 140 \mathrm{mg}$ in the morning and $60 \mathrm{mg}$ at night, 2 weeks on and 1 week off; oxaliplatin $130 \mathrm{mg} / \mathrm{m} 2$ intravenous drip d1, 3 weeks a cycle. Patients with disease control (complete response $(\mathrm{CR})+$ partial response $(\mathrm{PR})+$ stable disease (SD)) who could tolerate adverse reactions continue to take medication for 1 year, and will not take any other type of anti-tumour therapy until disease

\begin{tabular}{|c|c|c|}
\hline Dosage levels & Administration & Dosage \\
\hline 1 - standard dose & $12 \mathrm{mg} \mathrm{po}, \mathrm{qd}$ & $\begin{array}{l}12 \text { mg anlotinib } \\
\text { capsule, one } \\
\text { capsule }\end{array}$ \\
\hline $\begin{array}{l}2 \text { - reduced one } \\
\text { dose }\end{array}$ & $10 \mathrm{mg} \mathrm{po,} \mathrm{qd}$ & $\begin{array}{l}10 \mathrm{mg} \text { anlotinib } \\
\text { capsule, one } \\
\text { capsule }\end{array}$ \\
\hline $\begin{array}{l}3 \text { - reduced two } \\
\text { dose }\end{array}$ & $8 \mathrm{mg} \mathrm{po}, \mathrm{qd}$ & $\begin{array}{l}8 \text { mg anlotinib } \\
\text { capsule, one } \\
\text { capsule }\end{array}$ \\
\hline
\end{tabular}

po, oral; qd, four times per day.

progression occurred. Patients will be evaluated every 6 weeks (two cycles). Treatment will stop when the investigators conclude that the patients will be no longer good candidates to continue the presenting regimen or when the efficacy evaluation deem disease to be progressing.

\section{Dose adjustment protocol}

During administration, the investigator decide whether to perform dose adjustment, depending on the medication toxicity (graded according to NCI CTCAE V.4.0) and possible benefits. This study design two levels of dose adjustment(table 1). The treatment will be terminated if it is necessary to lower the two dose levels or more.

\section{Postpone dosing and dose adjustment}

The table 2 shows the recommended delay in medication administration and/or dosage changes when a therapyrelated toxicity occurs (platelet count drop, haemorrhage, liver dysfunction, proteinuria can be found in tables 3-6).

When neutrophils $\leq 1 \times 10^{9} /$ Lor platelets $\leq 50 \times 10^{9} / \mathrm{L}$, supportive treatment will be given, dose adjustment or

Table 2 The recommended delay in medication administration and dosage changes when a therapy-related toxicity occurs

\section{Adverse effect grade $\mathrm{NCl}$ CTCAE 4.0} Drug administration Dosage reduce

\begin{tabular}{|c|c|c|}
\hline Grade 0 to 2 & On time & No change \\
\hline Grade 3 & $\begin{array}{l}\text { Delay until adverse } \\
\text { effect grade is lower } \\
\text { than } 2^{*}\end{array}$ & $\begin{array}{l}\text { Reduce one dose } \\
\text { level }\end{array}$ \\
\hline Grade 4 & $\begin{array}{l}\text { Delay until adverse } \\
\text { effect grade is lower } \\
\text { than } 2^{*}\end{array}$ & $\begin{array}{l}\text { Reduce one dose } \\
\text { level. Terminate } \\
\text { treatment } \\
\text { if deemed } \\
\text { necessary }\end{array}$ \\
\hline
\end{tabular}

*If the delay does not recover after 3 weeks, the treatment should be terminated permanently.

CTCAE, Common Terminology Criteria for Adverse Events; NCl, National Cancer Institute. 
Table 3 The recommended delay in medication administration and dosage changes when a decrease in platelet count occurs

\begin{tabular}{|c|c|c|}
\hline Adverse effect grades & Dose adjusting protocol & Management \\
\hline $\begin{array}{l}\text { Grade } 1 \text { : PLT count } 100 \text { to } 75 \times \\
10^{9} / \mathrm{L}\end{array}$ & Maintain the original dose & Follow-up regularly \\
\hline \multirow[t]{2}{*}{ Grade 2: PLT count 75 to $50 \times 10^{9} / \mathrm{L}$} & $\begin{array}{l}\text { Delayed until adverse effect grade lower to } \\
<\text { Grade } 2 \text { in } 2 \text { weeks, resume original dose }\end{array}$ & $\begin{array}{l}\text { Repeat } \mathrm{RBC} \text { in } 2 \text { to } 3 \text { days; recommend } \\
\text { active treatment. RBC weekly during follow- } \\
\text { ups. }\end{array}$ \\
\hline & $\begin{array}{l}\text { Delayed until adverse effect grade lower to } \\
<\text { Grade } 2 \text { in } 3 \text { weeks, lower one dose level }\end{array}$ & $\begin{array}{l}\text { Repeat } \mathrm{RBC} \text { in } 2 \text { to } 3 \text { days; recommend } \\
\text { active treatment. RBC weekly during follow- } \\
\text { up. }\end{array}$ \\
\hline Grade 3: PLT count 50 to $25 \times 10^{9} / \mathrm{L}$ & $\begin{array}{l}\text { Delayed until adverse effect grade lower to } \\
<\text { Grade } 2 \text { in } 3 \text { weeks, lower one dose level }\end{array}$ & $\begin{array}{l}\text { Repeat } \mathrm{RBC} \text { in } 2 \text { to } 3 \text { days; recommend } \\
\text { active treatment. RBC weekly during follow- } \\
\text { up. }\end{array}$ \\
\hline Grade 4: PLT count $<25 \times 10^{9} / \mathrm{L}$ & Terminate therapy & $\begin{array}{l}\text { Daily RBC until adverse effect grade is } \\
\leq \text { Grade 2; actively give PLT transfusion. }\end{array}$ \\
\hline
\end{tabular}

PLT, platelet; RBC, red blood cell.

delay in medication administration will be considered when necessary. Table 3 shows the recommended delay in medication administration and/or dose changes when a decrease in platelet count occurs. Table 4 shows the recommended delay in medication administration and/ or dose changes after a bleeding event. Table 5 shows the recommended delay in medication administration and/or dose changes when liver function abnormalities (higher ALT, elevated AST or elevated total bilirubin) occur. Table 6 shows the recommended delay in medication administration and/or dose level changes when proteinuria occurs.

\section{Sample size calculation}

Literatures report that the objective response rate (ORR) rate of chemotherapy in patients with advanced gastric cancer is $30 \%$. We hypothesised that the ORR rate of anlotinib combined with SOX regimen in patients with stage IV gastric cancer is $40 \%, \alpha=0.05, \beta=0.8$, using PASS 11.0 software to estimate the best treatment group case number to be 136 . The final estimated sample size is 150 with an estimation of $10 \%$ loss of follow-up.

\section{Efficacy assessment indicators}

The main efficacy indicators were ORR, progression-free survival (PFS), disease control rate (DCR) and overall survival (OS). The ORR will be evaluated according to the efficacy evaluation criteria for solid tumours (RECIST 1.1). The DCR is defined as the percentage of CR, PR and $\mathrm{SD}$ and maintenance of the number of cases over 4 weeks in patients with evaluable efficacy. PFS is defined as the time from enrolment to the time when tumour progressed objectively. OS is defined as the time from enrolment to death of any cause. Subjects who lost to follow-up will be counted in days and usually documented according to the last follow-up time. For patients who discontinue the study without PD, the tumour evaluation results will be obtained during the follow-up period and recorded in the medical records until PD. Patients who survived until data analysis will be documented using the last follow-up when they are alive. The secondary efficacy indicator is safety indicator. The safety indicator is the incidence of adverse drug reactions and adverse events after administration. Adverse events refer to adverse medical events that occur in clinical trial patients after receiving the medications. In this study, an adverse event will be considered regardless if it is related to the therapy from the time when patients sign the informed consent form to 1 month after the end of treatment. Assessing the nature and determining the severity of adverse events will be conducted in accordance with CTCAE 4.0. To assess the adverse events and its causal relationship to therapy, the investigator will evaluate the possible associations between adverse events and trial medications. The following five criteria will be used to determine the results: the time of occurrence of adverse events coincide with the time of administration, adverse events are related to known adverse reactions of the medication, adverse events could not be explained by other reasons, adverse events disappeared after discontinuing therapy and adverse events are reproduced after medication administration. The results documented as positive,

Table 4 The recommended delay in medication administration and dosage changes after a bleeding event

\begin{tabular}{lll}
\hline Adverse effect grades & Dose adjusting protocol & Management \\
\hline Grade 1 & Maintain the original dose & Follow-up as planned \\
Grade 2 & Delayed until adverse effect grade lower to <Grade 2, lower one dose level & Active management \\
$\geq$ Grade 3 & Terminate therapy & Emergent intervention \\
\hline
\end{tabular}


Table 5 The recommended delay in medication administration and dosage changes when liver function abnormalities (higher ALT, elevated AST or elevated total bilirubin) occur

\begin{tabular}{|c|c|c|c|}
\hline \multicolumn{2}{|c|}{ Adverse effect grades } & \multirow{2}{*}{$\begin{array}{l}\text { Dose adjusting protocol } \\
\text { Maintain the original dose }\end{array}$} & \multirow{2}{*}{$\begin{array}{l}\text { Management } \\
\text { Follow-up as planned }\end{array}$} \\
\hline Grade 1 & & & \\
\hline \multirow[t]{2}{*}{ Grade 2} & $\begin{array}{l}\text { Normal } \\
\text { baseline }\end{array}$ & $\begin{array}{l}\text { Delayed until adverse effect grade lower to } \\
<\text { Grade } 2 \text { in } 3 \text { weeks, lower one dose level }\end{array}$ & $\begin{array}{l}\text { Actively reserve and monitor hepatic function } \\
\text { weekly }\end{array}$ \\
\hline & $\begin{array}{l}\text { Abnormal } \\
\text { baseline }\end{array}$ & Maintain the original dose & $\begin{array}{l}\text { Actively reserve and monitor hepatic function } \\
\text { weekly }\end{array}$ \\
\hline \multicolumn{2}{|l|}{ Grade 4} & Terminate therapy & $\begin{array}{l}\text { Actively reserve and monitor liver function twice } \\
\text { weekly until adverse effect lower to }<\text { Grade } 2 \text { or can } \\
\text { be explained }\end{array}$ \\
\hline
\end{tabular}

ALT, alanine aminotransferase; AST, aspartate aminotransferase.

relevant and possibly related will be deemed to be adverse reactions(table 7). The incidence of adverse reactions will be calculated accordingly. To record, process and report adverse events, the investigator will document any adverse events. Records of adverse events will include a description of adverse events and all related symptoms, time of occurrence, severity, duration, measures taken, results and final outcomes. The reporting methods and treatment measures for severe adverse events will classify as severe adverse events if they meet one or more of the following criteria: death, life-threatening (eg, immediate risk of death), prolonged hospitalisation or hospitalisation, permanent or severe disability, congenital malformations or defects, some events that has not yet caused death, danger to life or hospitalisation; but will consider a severe adverse event by a physician if they cause harm to the patient or require medication or surgical treatment to avoid the above situation. For any severe adverse events during the clinical trial, the investigator will file a report of severe adverse events within 24 hours and report in writing to the Ethics Committee, the superior authorities and the sponsor. The written report will include the time, severity, duration, measures taken and outcomes of serious adverse events. The patients' quality of life will also be evaluated by the Quality of Life QuestionnaireCancer (QLQ-C30) and the site-specific module for gastric cancer (QLQ-STO22) ${ }^{19} 20$

\section{Data collection and observation indicators}

The baseline data (Collected medical history and demographics including patient gender, age and contact number; Detailed medical history, treatment history, ECOG score, body mass index (BMI); 12 ECG (pay special attention to QTc); red blood cell (RBC), urinalysis (UA), stool analysis (including faecal occult blood); comprehensive metabolic panel (CMP) including ALT, AST, TBIL, directbilirubin, indirectbilirubin, bloodurea nitrogen, creatinine, electrolytes (K+, Na+, CL-, Ca2+); Coagulation test (Prothrombin time (PT), activated partial thromboplastin time (APTT), thrombin time (TT), fasting blood glucose (FBG), D-dimer, international normalized ratio (INR)); Serum carcinoembryonic antigen (CEA), Carbohydrate antigen199 (CA199)) will be recorded. Premedication imaging assessment test (CT/MRI) included enhanced CT or MRI of the chest, abdomen and pelvis will be completed within 21 days before medication

Table 6 The recommended delay in medication administration and dosage changes when proteinuria occurs

\begin{tabular}{|c|c|c|}
\hline Adverse effect grade & Drug administration & Management \\
\hline $\begin{array}{l}\text { Grade } 2 \text { : UA shows protein }++ \\
\text { or higher, but } 24 \text { hours protein } \\
\text { quantity }>2 \mathrm{~g} \text { and }<3.5 \mathrm{~g}\end{array}$ & $\begin{array}{l}\text { Delayed until adverse effect grade lower } \\
\text { to }<\text { Grade } 2 \text { in } 3 \text { weeks, lower one dose } \\
\text { level }\end{array}$ & $\begin{array}{l}\text { Active treatment; consult nephrologist } \\
\text { when necessary, terminate treatment when } \\
\text { experiences adverse effect for the third time }\end{array}$ \\
\hline $\begin{array}{l}\text { Grade } 3: 24 \text { hours urine protein } \\
\geq 3.5 \mathrm{~g}\end{array}$ & $\begin{array}{l}\text { Delayed until adverse effect grade lower } \\
\text { to }<\text { Grade } 2 \text { in } 3 \text { weeks, lower one dose } \\
\text { level }\end{array}$ & $\begin{array}{l}\text { Active treatment; consult nephrologist } \\
\text { when necessary, terminate treatment when } \\
\text { experiences adverse effect for the third time }\end{array}$ \\
\hline
\end{tabular}

UA, urinalysis. 
Table 7 The possible evaluation of the causal relationship between adverse events and trial medications

\begin{tabular}{lccccc}
\hline & $\mathbf{1}$ & $\mathbf{2}$ & $\mathbf{3}$ & $\mathbf{4}$ & $\mathbf{5}$ \\
\hline Definitely relevant & + & + & + & + & + \\
Very likely & + & + & + & + & $?$ \\
Possible related & + & + & \pm & \pm & $?$ \\
May not be relevant & + & -- & \pm & \pm & $?$ \\
Definitely irrelevant & -- & -- & -- & -- & -- \\
\hline
\end{tabular}

?, possibly related; --, negative; +, positive; \pm , hard to be positive or negative.

administration. Positronemission tomography examination will not be performed as a routine imaging assessment. All suspected lesions will be evaluated by imaging test. When patients are suspected of brain metastases or showed clinical symptoms/signs, a CT or MRI of the head will be performed. Female patients of childbearing age will be also tested with hCG (humanchorionic gonadotropin) for pregnancy.

During treatment, adverse effects including nausea, vomiting, diarrhoea and bloating will be documented. At end of each cycle, the following tests will be taken: EKG (special attention to QTc), CBC, UA, stool analysis, CMP, coagulation function tests, cardiac enzymes (creatine kinase (CK), CK-MB) and troponin will be added if chest pain, palpitations and other relevant symptoms. When urine protein ++ or above, 24 hours urine protein quantitation will be performed within 1 week. Efficacy evaluation will perform every two cycles (including imaging (CT/MRI) + serum CEA, CA199 test). Thyroid function (T3, T4, FT3, FT4, thyroid-stimulatinghormone) will be tested every two cycles. The QLQ-C30 and QLQ-STO22 of the patients will also be collected.

\section{Follow-up}

The follow-up phase will start after the last medication administration. Patients with unrecovered adverse effects will treat and follow-up closely until they recover to NCI CTCAE V4.0 first degree or recover fully. Regular follow-up will be scheduled once every two cycles until patients' death, the cause and date of death will be documented to calculate the OS. Follow-up tests include: BMI, EKG, RBC, CMP, UA, stool analysis, electrolytes, tumour markers (serum CEA, CA199), coagulation function, thyroid function, image test (enhanced CT or MRI of the chest, abdomen and pelvis). Survival state will be collected and analysed. In addition, the quality of life will also be assessed.

\section{Statistics}

All statistical analyses will be analysed using SPSS 22.0 statistical analysis software. The data will be statistically described by the mean \pm SD or median (minimum, maximum). All statistical tests will be performed as twosided tests with a $p$ value $\leq 0.05$ that will be considered statistically significant, and a $95 \%$ CI will be used for the
CI. The measured data will compare with baseline values obtained before treatment, and the paired t-test will be used to achieve this analysis. Changes before and after treatment in each group will compare by analysis of variance or rank-sum test. The counting data will be statistically described using frequency (composition ratio). Changes before and after treatment will be tested by the $\chi 2$ test for an exact probability or non-parametric test.

\section{Definition of the analytical data set}

For the full analysis set, efficacy analyses will be conducted of all cases with at least one medication administration, using the intention analysis method. For the per-protocol set, all cases completed therapy for at least 6 weeks and followed the protocol with good compliance, or do not use medication during the trial or completed the case report forms will be analysed while evaluating drug efficacy. The missing data will not be filled. For the safety analysis set, all patients enrolled in the study received at least one session of therapy, with post-medication safety records will be included in the safety analysis set. This data set will be used for safety analysis.

Baseline data will be analysed as the full analysis set, and all efficacy indexes will be analysed with the full analysis and compliance sets; the safety analysis use the safety analysis set. For dropout analysis, descriptive analysis will be mainly used. The list describes the specific causes of dropout, and the total rate and rate of dropout due to adverse effects will be calculated. For validity analysis, the product limit method will be used to estimate the PFS and OS. The 25\%, 50\% (median), $75 \%$ PFS and PFS and OS at different times after treatment started will be calculated using collected data. For safety analysis, descriptive statistical analysis will be the main method. The list describes the adverse effects that occurred in this trial. The analysis also describes abnormal test results that will be normal before treatment and its correlation between treatment and the changed abnormal lab test results.

\section{Ethics and dissemination}

Ethics approval has been obtained from the Ethics Committee at the First Affiliated Hospital (Xijing Hospital) of Fourth Military Medical University (KY20192111-F-1). It is the investigator's responsibility to provide each study candidate or his/her legal representative with a complete and comprehensive explanation of the purpose, process and possible benefits and risks of the study, to obtain the signed written informed consent forms before enrolling (see the details of the informed consent form in online supplementary file). The present study protocol was prepared in accordance of the Standard Protocol Items: Recommendations for Intervention Trials statement. Subjects will be informed that they have the right to withdraw from the study at any time, and informed consent will be retained as a clinical study document for future reference. The subject's personal privacy and data confidentiality will be protected during the study. The results of this study will be disseminated at 
several research conferences and as published articles in peer-reviewed journals.

\section{Data management}

Investigators will record the relevant data of each patient in the research medical record in a timely and true manner. The researcher or his authorised personnel will fill in the relevant information in the case report form, and ensure that the content entered is consistent with the research medical record. To ensure patient privacy, codes will be used instead of the patient's name. After completion of the study, the case report form will be kept by the sponsor and the research facility and it will be ensured that data will be correctly entered. Key investigators, data administrators, statisticians and auditors will determine the data set for analysis. Under most circumstances, the determined data file will no longer be changed.

\section{Patient and public involvement}

Patients or the public will not be involved in the design, or conduct, or reporting or dissemination of our research.

Contributors GJ and JW conceived and designed the study. JW wrote the paper. DXW and LM checked the statistical methods. All authors read and approved the manuscript and agree to be accountable for all aspects of the research in ensuring that the accuracy or integrity of any part of the work are appropriately investigated and resolved.

Funding This work was supported by the grant from the National Natural Science Foundation of China (Key Programme 81502401 and 31670828).

Competing interests None declared.

Patient and public involvement Patients and/or the public were not involved in the design, or conduct, or reporting or dissemination plans of this research.

Patient consent for publication Not required.

Provenance and peer review Not commissioned; externally peer reviewed.

Open access This is an open access article distributed in accordance with the Creative Commons Attribution Non Commercial (CC BY-NC 4.0) license, which permits others to distribute, remix, adapt, build upon this work non-commercially, and license their derivative works on different terms, provided the original work is properly cited, appropriate credit is given, any changes made indicated, and the use is non-commercial. See: http://creativecommons.org/licenses/by-nc/4.0/.

ORCID iD

Gang Ji http://orcid.org/0000-0001-7500-7192

\section{REFERENCES}

1 Jayavelu ND, Bar NS. Metabolomic studies of human gastric cancer: review. World J Gastroenterol 2014;20:8092-101.

$2 \mathrm{He} \mathrm{J}$, Zhao P, Chen WQ. Chinese cancer registry annual report. Beijing: Academy of Military Medical Science Press, 2012.

3 Bilici A. Treatment options in patients with metastatic gastric cancer: current status and future perspectives. World J Gastroenterol 2014;20:3905-15.

4 Davidson M, Chau I. Multimodality treatment of operable gastric and oesophageal adenocarcinoma: evaluating neoadjuvant, adjuvant and perioperative approaches. Expert Rev Anticancer Ther 2018;18:327-38.

5 Wagner AD, Syn NL, Moehler M, Unverzagt S, Grothe W, et al. Chemotherapy for advanced gastric cancer. Cochrane Database Syst Rev 2017;8:CD004064.

6 Zhou M, Chen X, Zhang H, et al. China national medical products administration approval summary: anlotinib for the treatment of advanced non-small cell lung cancer after two lines of chemotherapy. Cancer Commun 2019;39:36.

7 Yang S, Zhang Z, Wang Q. Emerging therapies for small cell lung cancer. J Hematol Oncol 2019;12:47.

8 Sun Y, Du F, Gao M, et al. Anlotinib for the treatment of patients with locally advanced or metastatic medullary thyroid cancer. Thyroid 2018;28:1455-61.

9 Ruan X, Shi X, Dong Q, et al. Antitumor effects of anlotinib in thyroid cancer. Endocr Relat Cancer 2019;26:153-64.

10 Han B, Li K, Wang Q, et al. Effect of Anlotinib as a third-line or further treatment on overall survival of patients with advanced non-small cell lung cancer: the ALTER 0303 phase 3 randomized clinical trial. JAMA Oncol 2018;4:1569-75.

11 Han B, Li K, Zhao Y, et al. Anlotinib as a third-line therapy in patients with refractory advanced non-small-cell lung cancer: a multicentre, randomised phase II trial (ALTER0302). Br J Cancer 2018;118:654-61.

12 Power DG, Kelsen DP, Shah MA. Advanced gastric cancer--slow but steady progress. Cancer Treat Rev 2010;36:384-92.

13 Perrimon N. The torso receptor protein-tyrosine kinase signaling pathway: an endless story. Cell 1993;74:219-22.

$14 \mathrm{He} \mathrm{C}$, Wu T, Hao Y. Anlotinib induces hepatocellular carcinoma apoptosis and inhibits proliferation via ERK and Akt pathway. Biochem Biophys Res Commun 2018;503:3093-9.

15 Shen G, Zheng F, Ren D, et al. Anlotinib: a novel multi-targeting tyrosine kinase inhibitor in clinical development. J Hematol Oncol 2018;11:120.

16 Lin B, Song X, Yang D, et al. Anlotinib inhibits angiogenesis via suppressing the activation of VEGFR2, PDGFR $\beta$ and FGFR1. Gene 2018;654:77-86.

17 Xie C, Wan X, Quan H, et al. Preclinical characterization of anlotinib, a highly potent and selective vascular endothelial growth factor receptor-2 inhibitor. Cancer Sci 2018;109:1207-19.

18 Taurin S, Yang C-H, Reyes M, et al. Endometrial cancers harboring mutated fibroblast growth factor receptor 2 protein are successfully treated with a new small tyrosine kinase inhibitor in an orthotopic mouse model. Int J Gynecol Cancer 2018;28:152-60.

19 Kobayashi D, Kodera Y, Fujiwara M, et al. Assessment of quality of life after gastrectomy using EORTC QLQ-C30 and STO22. World J Surg 2011;35:357-64.

20 Ito Y, Yoshikawa T, Fujiwara M, et al. Quality of life and nutritional consequences after aboral pouch reconstruction following total gastrectomy for gastric cancer: randomized controlled trial CCG1101. Gastric Cancer 2016;19:977-85. 